\title{
Author Reply
}

\section{Didier Mazel*}

*Unité Plasticité du Génome Bactérien- CNRS URA 2171, Department Génomes et Génétiqu, Institut Pasteur, 25 rue du Dr Roux, 75724 Paris, France. E-mail: mazel@pasteur.fr

In their letter, Hall and co-authors argue that there is no difference between integrons carried by mobile genetic elements and those integrated into the chromosomes that are sometimes referred to as superintegrons. For a detailed account of my arguments relating to this proposed division, I refer readers to my recent Review ${ }^{1}$.

Although I agree that all integrons share basic defining properties (for example, possession of an intI gene and the attI site), in my opinion, it is not clear that all integrons have the same structure and mechanism. As discussed in my recent Review article, I argue that superintegrons have properties that distinguishes them from other plasmid- or transposon-associated integrons ${ }^{1}$. Of course, further studies will be required to ascertain whether or not structural differences are linked to functional differences of biological relevance. Finally, on a more general note, many aspects of integron biology are not yet understood; therefore, at present, I take the view that it is too early to draw general conclusions regarding a universal integron structure and mechanism.

1. Mazel, D. Integrons: agents of bacterial evolution. Nature Rev. Microbiol. 4, 608620 (2006). 